\title{
Mapping the Photoresponse of Quantum-Dot-Based Photon-Number-Resolving Detectors
}

\author{
Brecca T. Bettcher, ${ }^{1}$ Trevor R. Geerdts, ${ }^{2}$ Connor P. Govin, ${ }^{3}$ \\ and Eric J. Gansen ${ }^{4}$ \\ Physics Department, University of Wisconsin-La Crosse \\ La Crosse, WI, USA
}

Author responses to review comments:

Comment 1. Usually, the photon number resolving capabilities for a single photon detector are presented by dispalying a typical temporal pulse shape and then a pulse height distribution spectrum. Does the output of the QDOGFET detector provide such timing information?

Response: Yes, high-quality response histograms like the one the reviewer suggests have been acquired using QDOGFETs and their photon-number-resolving qualities demonstrated. Such histograms have been presented numerous times in the references sited in the introductory section of the proceedings. While such histograms are gathered as an intermediate step in acquiring the response maps that are the subject of this work, they are not included in order to keep the paper brief and focused.

Comment 2. In Figure 2 (b), the gain of the amplifier is $\sim 100$ and the laser is providing $15 \mathrm{~ns}$ wide pulses. The GBW product required seems quite high could you provide some additonal details on the analog detection circuit?

Response: While the laser pulses are $15 \mathrm{~ns}$ long, the response time of the amplifier is much longer. Keep in mind that the QDOGFET exhibits a persistent response to each pulse of light that lasts for much longer than the pulse duration. As a result, the amplifier doesn't need to respond on the 10-ns timescale. The 100x amplifier had a (LPF) bandwidth of $100 \mathrm{kHz}$ for all the measurements presented in this work.

Comment 3. The data presented is very compelling and the analysis is very thorough. The physics of the transconductance of the channel and pinch off effect is correct.

Response: Here, here.

Comment 4. The left hand side of Figure 1 (a) does not match the rest of the figure. I could not quite interpret it.

Response: The text and figure have been revised in order to make the relationship between parts (a) and (b) of the figure more clear. 